\title{
The order of $L^{1}$-approximation by elements of the disc algebra
}

\section{Vilmos Totik ${ }^{1}$}

Received: 17 September 2021 / Accepted: 21 September 2021 / Published online: 9 November 2021 (c) The Author(s) 2021

\section{Abstract}

We prove that the order of $L^{1}$-approximation by elements of the disc algebra given by Khavinson, Pérez-González and Shapiro is precise.

Let $\Delta$ be the unit disc, $\mathbf{T}$ its boundary and consider the disk algebra $\mathcal{A}$ of those continuous functions on $\bar{\Delta}$ that are holomorphic in $\Delta$. In $\mathcal{A}$ the norm is the supremum norm

$$
\|f\|_{\infty}=\sup _{t}\left|f\left(e^{i t}\right)\right|,
$$

but we shall also consider the $L^{1}$ norm given by

$$
\|f\|_{1}=\frac{1}{2 \pi} \int_{0}^{2 \pi}\left|f\left(e^{i t}\right)\right| d t .
$$

In connection with approximation in $L^{1}$-norm by elements of a uniform algebra D. Khavinson, F. Pérez-González and H. Shapiro proved the following theorem (see [2, Theorem 3.3]).

Theorem Let $f$ be a continuous function on $\mathcal{T}$ with $\|f\|_{\infty}=1$. Assume there exists an $H^{1}$-function $G$ such that

$$
\|f-G\|_{1} \leq \varepsilon
$$

Then there exists a function $G^{*}$ in the disk algebra $\mathcal{A}$ such that $\left\|G^{*}\right\|_{\infty} \leq 1$ and

$$
\left\|f-G^{*}\right\|_{1} \leq C \varepsilon \log \frac{1}{\varepsilon}
$$

$凶$ Vilmos Totik

totik@math.u-szeged.hu

1 MTA-SZTE, Analysis and Stochastics Research Group Bolyai Institute, University of Szeged, Szeged Aradi v. tere 1, 6720, Hungary 
where $C$ is a constant independent of $f$.

See [2] for the motivation of this result and its connection to a theorem of Hoffman and Wermer on homomorphisms of uniform algebras.

The authors of [2] also verified that in (1) the bound $C \varepsilon \log \frac{1}{\varepsilon}$ cannot be replaced by $C \varepsilon$ ( [2, Theorem 3.4]), but the problem if the order $O\left(\varepsilon \log \frac{1}{\varepsilon}\right)$ in (1) can be improved at all, i.e., if it is precise or not, remained open and stated explicitly in Remark (i) in [2]. That problem was communicated to us by D. Khavinson [1]. In this note we prove that the stated order is, indeed, precise.

Theorem 1 There is a constant $c>0$ with the property that for every sufficiently small $\varepsilon>0$ there is a continuous function $f=f_{\varepsilon},\|f\|_{\infty}=1$, such that

$$
\|f-G\|_{1} \leq \varepsilon
$$

for some $G \in \mathcal{A}$, but for any $G^{*}$ in $\mathcal{A}$ with $\left\|G^{*}\right\|_{\infty} \leq 1$ we have

$$
\left\|f-G^{*}\right\|_{1} \geq c \varepsilon \log \frac{1}{\varepsilon} .
$$

Proof It will be convenient to verify the claim with $\varepsilon$ replaced by $\varepsilon^{2}$.

Let

$$
u(z)+i v(z)=\frac{\varepsilon^{2}}{1+\varepsilon-z},
$$

where $u(z)$ and $v(z)$ are real. Using that

$$
(1+\varepsilon-\cos t)^{2}+\sin ^{2} t=\varepsilon^{2}+4(1+\varepsilon) \sin ^{2}(t / 2),
$$

for $z=e^{i t}$ we have ${ }^{1}$

$$
\Re \frac{1}{1+\varepsilon-z}=\frac{1+\varepsilon-\cos t}{(1+\varepsilon-\cos t)^{2}+\sin ^{2} t} \sim \begin{cases}1 / \varepsilon & \text { if }|t| \leq \varepsilon, \\ 1+\varepsilon / t^{2} & \text { if } \varepsilon \leq|t| \leq \pi,\end{cases}
$$

while

$$
\left|\Im \frac{1}{1+\varepsilon-z}\right|=\frac{|\sin t|}{(1+\varepsilon-\cos t)^{2}+\sin ^{2} t} \sim \begin{cases}|t| / \varepsilon^{2} & \text { if }|t| \leq \varepsilon \\ 1 /|t| & \text { if } \varepsilon \leq|t| \leq \pi / 2 \\ \pi-|t| & \text { if } \pi / 2 \leq|t| \leq \pi\end{cases}
$$

Indeed, these are easy consequences of the inequality

$$
\frac{2}{\pi} u \leq \sin u \leq u, \quad 0 \leq u \leq \pi / 2,
$$

\footnotetext{
$1_{1}$ In what follows $A \sim B$ means that $A / B$ lies in between two positive absolute constants, and $A \preceq B$ and $B \succeq A$ stand for $A / B$ being bounded.
} 
i.e. of

$$
\sin u \sim u, \quad 0 \leq u \leq \pi / 2
$$

For example, for $z=e^{i t}, \varepsilon \leq|t| \leq \pi(0<\varepsilon \leq 1)$, we obtain

$$
\begin{aligned}
\Re \frac{1}{1+\varepsilon-z} & =\frac{1+\varepsilon-\cos t}{(1+\varepsilon-\cos t)^{2}+\sin ^{2} t}=\frac{2 \sin ^{2}(t / 2)+\varepsilon}{\varepsilon^{2}+4(1+\varepsilon) \sin ^{2}(t / 2)} \\
& \sim \frac{t^{2}+\varepsilon}{\varepsilon^{2}+t^{2}} \sim \frac{t^{2}+\varepsilon}{t^{2}}=1+\varepsilon / t^{2}
\end{aligned}
$$

as was claimed above.

The preceding relations show that

$$
u\left(e^{i t}\right) \sim \begin{cases}\varepsilon & \text { if }|t| \leq \varepsilon \\ \varepsilon^{2}+\varepsilon^{3} / t^{2} & \text { if } \varepsilon \leq|t| \leq \pi\end{cases}
$$

while

$$
\left|v\left(e^{i t}\right)\right| \sim \begin{cases}|t| & \text { if }|t| \leq \varepsilon \\ \varepsilon^{2} /|t| & \text { if } \varepsilon \leq|t| \leq \pi / 2 \\ \varepsilon^{2}(\pi-|t|) & \text { if } \pi / 2 \leq|t| \leq \pi\end{cases}
$$

Therefore, if we set $F_{\varepsilon}=F(z)=\exp \left(\varepsilon^{2} /(1+\varepsilon-z)\right),|z| \leq 1$, then for small $\varepsilon$

$$
\begin{aligned}
\Re F\left(e^{i t}\right)-1 & =e^{u\left(e^{i t}\right)} \cos v\left(e^{i t}\right)-1 \geq\left(1+u\left(e^{i t}\right)\right) \cos v\left(e^{i t}\right)-1 \\
& =u\left(e^{i t}\right) \cos v\left(e^{i t}\right)-2 \sin ^{2}\left(v\left(e^{i t}\right) / 2\right)>0 .
\end{aligned}
$$

This is so, because we subtract from a term $\sim \varepsilon(|t| \leq \varepsilon) \operatorname{resp} \succeq \varepsilon^{3} / t^{2}(|t| \geq \varepsilon)$ a term that is at most $\preceq \varepsilon^{2}$ resp. $\preceq \varepsilon^{4} / t^{2}$. Now the preceding inequality implies in view of the maximum principle that $\Re F(z)-1$ is positive in the unit disk.

Let $f_{\varepsilon}=f=F /|F|$, for which we have for small $\varepsilon$

$$
\begin{aligned}
\|f-F\|_{1} & =\left\|\frac{F}{|F|}(|F|-1)\right\|_{1}=\||F|-1\|_{1}=\frac{1}{2 \pi} \int_{-\pi}^{\pi}\left(e^{u\left(e^{i t}\right)}-1\right) d t \\
& \sim \int_{-\pi}^{\pi} u\left(e^{i t}\right) d t \sim \varepsilon^{2},
\end{aligned}
$$

where we used that $u \leq e^{u}-1 \leq 2 u$ provided $0 \leq u \leq 1 / 2$ (cf. (2)).

Note that $F$ is in the disk algebra and $f$ is a continuous function with $\|f\|_{\infty}=1$. Now let $G^{*} \in \mathcal{A},\left\|G^{*}\right\|_{\infty} \leq 1$, be any function. We are going to show that

$$
\left\|f-G^{*}\right\|_{1} \geq c \varepsilon^{2} \log \frac{1}{\varepsilon}
$$


with some $c>0$ independent of $\varepsilon$, and that will prove the theorem (with $\varepsilon$ replaced by $\varepsilon^{2}$ and $f_{\alpha \varepsilon}$ resp. $F_{\alpha \varepsilon}$ replacing $f$ resp. $G$ in it, where $\alpha$ is a constant for which $\left\|f_{\alpha \varepsilon}-F_{\alpha \varepsilon}\right\|_{1} \leq \varepsilon^{2}$; see (4)).

For the $L^{1}$ distance of $F$ and $G^{*}$ we have

$$
\left\|F-G^{*}\right\|_{1} \leq\|F-f\|_{1}+\left\|f-G^{*}\right\|_{1} \leq C_{1} \varepsilon^{2}+\left\|f-G^{*}\right\|_{1} .
$$

The real part of

$$
g_{1}(z):=1-G^{*}(z)+i \Im G^{*}(0)
$$

is clearly nonnegative. Now to the pairs $g_{1}(z)$ and $g_{2}(z):=F(z)-1$ with nonnegative real part in $\Delta$ and with imaginary part $=0$ at the origin we can apply the "reverse triangle inequality"

$$
\left\|g_{1}\right\|_{1}+\left\|g_{2}\right\|_{1} \leq C_{0}\left\|g_{1}+g_{2}\right\|_{1},
$$

proved in [2, Lemma 3.5], where $C_{0}$ is an absolute constant. This yields

$$
\begin{aligned}
\|F-1\|_{1} & \leq\left\|g_{1}\right\|_{1}+\left\|g_{2}\right\|_{1} \leq C_{0}\left\|(F-1)+\left(1-G^{*}+i \Im G^{*}(0)\right)\right\|_{1} \\
& =C_{0}\left\|F-G^{*}+i \Im G^{*}(0)\right\|_{1} \leq C_{0}\left(\left\|F-G^{*}\right\|_{1}+\left|i \Im G^{*}(0)\right|\right) .
\end{aligned}
$$

On the right

$$
\left|i \Im G^{*}(0)\right|=\left|\Im\left(F-G^{*}\right)(0)\right| \leq\left\|F-G^{*}\right\|_{1},
$$

so, in view of (6),

$$
\|F-1\|_{1} \leq 2 C_{0}\left\|F-G^{*}\right\|_{1} \leq 2 C_{0} C_{1} \varepsilon^{2}+2 C_{0}\left\|f-G^{*}\right\|_{1}
$$

follows. Since on the left

$$
\begin{aligned}
\|F-1\|_{1} \geq\|\Im F\|_{1} & =\frac{1}{2 \pi} \int_{-\pi}^{\pi} e^{u\left(e^{i t}\right)}\left|\sin v\left(e^{i t}\right)\right| d t \geq \frac{1}{2 \pi} \int_{-\pi}^{\pi}\left|\sin v\left(e^{i t}\right)\right| d t \\
& \succeq \frac{1}{2 \pi} \int_{\varepsilon}^{\pi / 2}\left|v\left(e^{i t}\right)\right| d t \sim \varepsilon^{2} \log \frac{1}{\varepsilon}=\frac{1}{2} \varepsilon^{2} \log \frac{1}{\varepsilon^{2}}
\end{aligned}
$$

(where, for the $\sim$ relation we used (3)), the inequality (5) follows from (7) for all sufficiently small $\varepsilon$.

Data sharing is not applicable to this article as no datasets were generated or analyzed during the current study. ${ }^{2}$

\footnotetext{
2 This statement was requested to be included during the submission process.
} 
Funding Open access funding provided by University of Szeged.

\section{Declarations}

Conflict of interest There is no conflict of interest.

Open Access This article is licensed under a Creative Commons Attribution 4.0 International License, which permits use, sharing, adaptation, distribution and reproduction in any medium or format, as long as you give appropriate credit to the original author(s) and the source, provide a link to the Creative Commons licence, and indicate if changes were made. The images or other third party material in this article are included in the article's Creative Commons licence, unless indicated otherwise in a credit line to the material. If material is not included in the article's Creative Commons licence and your intended use is not permitted by statutory regulation or exceeds the permitted use, you will need to obtain permission directly from the copyright holder. To view a copy of this licence, visit http://creativecommons.org/licenses/by/4.0/.

\section{References}

1. Khavinson, D.: Personal communication

2. Khavinson, D., Pérez-González, P., Shapiro, H.: Approximation in $L^{1}$-norm by elements of a uniform algebra. Constr. Approx. 14, 401-410 (1998)

Publisher's Note Springer Nature remains neutral with regard to jurisdictional claims in published maps and institutional affiliations. 